\title{
Successful Use of Microporous Polytetrafluoroethylene Flexible Thin Sheets in NASA's OSIRIS-REx Mission
}

\author{
Michael K. Choi, ${ }^{1}$ Brent J. Bos, ${ }^{2}$ and June L. Tveekrem ${ }^{3}$ \\ NASA Goddard Space Flight Center, Greenbelt, MD 20771 \\ Christian d'Aubigny, ${ }^{4}$ Bashar Rizk, ${ }^{5}$ and Dante S. Lauretta ${ }^{6}$ \\ Lunar and Planetary Laboratory, University of Arizona, Tucson, AZ 85721 \\ and \\ The OSIRIS-REx Team
}

Microporous black polytetrafluoroethylene (PTFE) flexible thin sheets are successfully flown as solar diffusers on NASA's Origins, Spectral Interpretation, Resource Identification, and Security-Regolith Explorer (OSIRIS-REx) spacecraft. They serve as multilayer insulation (MLI) blanket outer covers for the arm of the Touch And Go Sample Acquisition Mechanism (TAGSAM), the sunshade of the OSIRIS-REx Camera Suite (OCAMS) PolyCam imager, and the motor riser of the OCAMS SamCam imager. Additionally, microporous white PTFE flexible thin sheets are successfully flown as a MLI blanket outer cover with a low ratio of absorptance to emittance for the Regolith X-ray Imaging Spectrometer (REXIS). For ground testing, microporous black and white PTFE flexible thin sheets were successfully used as optical targets of the Touch And Go Camera System (TAGCAMS) NavCam imagers in the flight system thermal vacuum test.

\section{Nomenclature}

$\begin{array}{ll}A U & =\text { astronomical unit } \\ B R D F & =\text { bidirectional reflectance distribution function } \\ C C D & =\text { charge coupled device } \\ \text { OSIRIS-REX } & =\text { Origins, Spectral Interpretation, Resource Identification, and Security-Regolith Explorer } \\ \text { FOV } & =\text { field of view } \\ G B K & =\text { germanium coated black Kapton } \\ M L I & =\text { multilayer insulation } \\ \text { OCAMS } & =\text { OSIRIS-REx Camera Suite } \\ \text { PTFE } & =\text { polytetrafluoroethylene } \\ \text { REXIS } & =\text { Regolith X-ray Imaging Spectrometer } \\ \text { TAG } & =\text { Touch And Go } \\ \text { TAGCAMS } & =\text { Touch And Go Camera System } \\ \text { TAGSAM } & =\text { Touch And Go Sample Acquisition Mechanism } \\ T V A C & =\text { thermal vacuum }\end{array}$

\section{Introduction}

NASA's OSIRIS-REx spacecraft was successfully launched on September 8, 2016. Its mission is to travel to nearEarth asteroid (101955) Bennu (Figure 1), study it in detail, and bring back a pristine sample to Earth for scientific

\footnotetext{
${ }^{1}$ Senior Aerospace Engineer, Heat Transfer, AIAA Associate Fellow.

2 Optical Engineer.

${ }^{3}$ Optical Engineer.

${ }^{4}$ OCAMS Lead Optical Engineer.

${ }^{5}$ OCAMS Senior Staff Scientist.

${ }^{6}$ OSIRIS-REx Principal Investigator.
} 
analyses. ${ }^{1}$ The spacecraft arrived at Bennu on December 3, 2018. It will spend approximately one year surveying the asteroid, with the goal of selecting a site for sample collection. It is scheduled to return a sample to Earth in September 2023.

The spacecraft's Touch And Go Sample Acquisition Mechanism (TAGSAM) has an arm, motors, frangibolts, nitrogen bottles, gas-handling system, and sampler head. ${ }^{2}$ Touch And Go (TAG) sampling occurs at a heliocentric distance of 1.25 to 1.36 astronomical units (AU). ${ }^{3}$ Figure 2 shows an artist's impression of OSIRIS-REx, with the TAGSAM deployed. During the TAG event, the TAGSAM is exposed to the Sun continuously for as much as five days. The TAGSAM arm is insulated with multilayer insulation (MLI) blankets from the extreme thermal environments in flight.

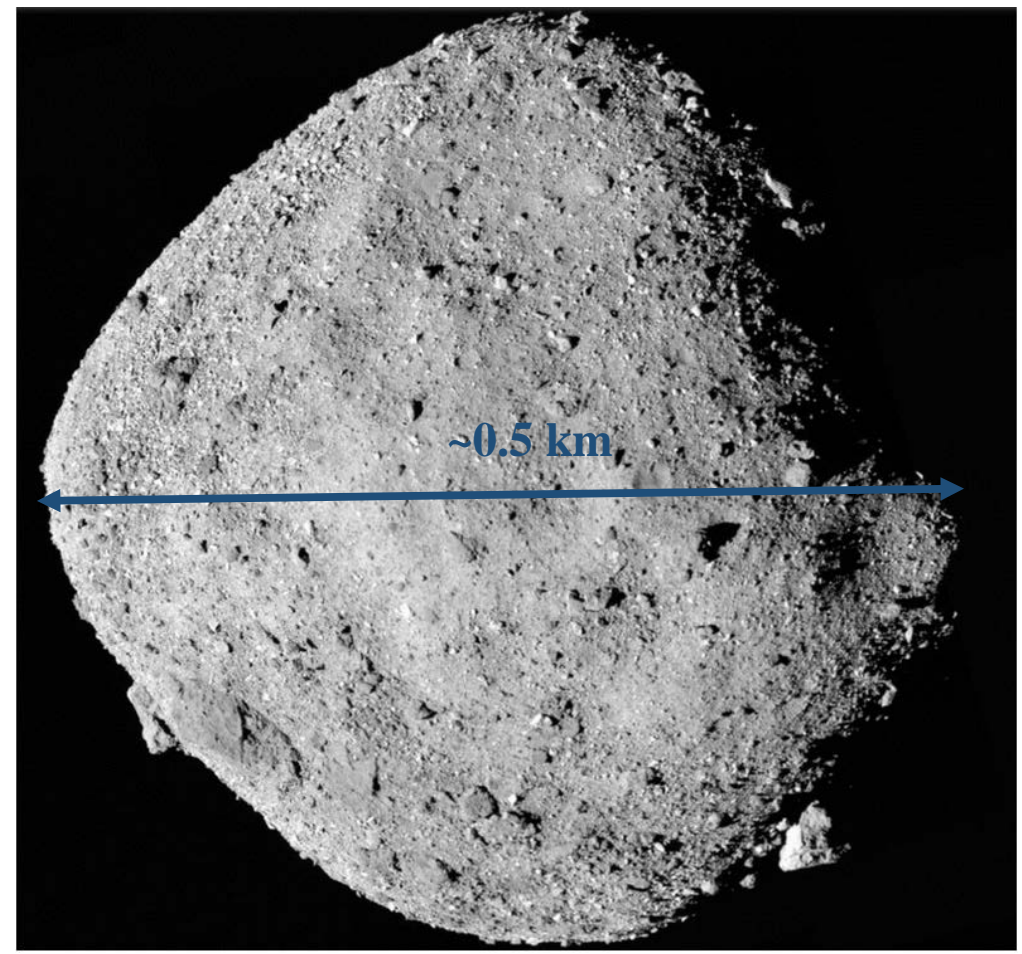

Fig. 1. Image of asteroid Bennu obtained by OSIRIS-REx on December 2, 2018 (Credit: NASA/Goddard/University of Arizona).

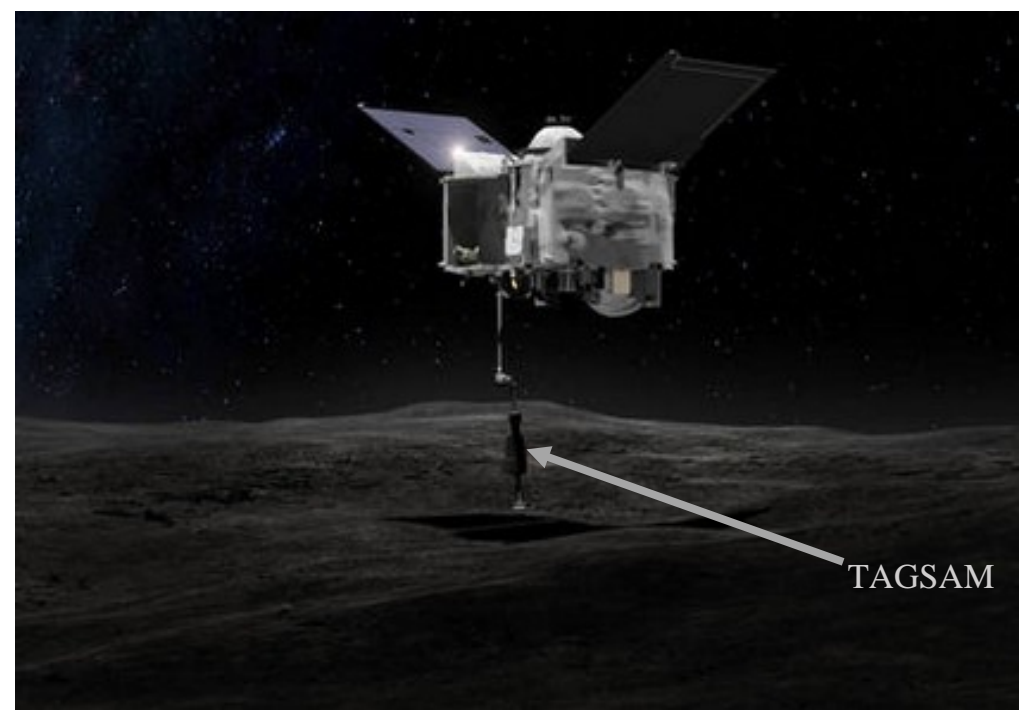

Fig. 2. Artist's impression of OSIRIS-REx during TAG at asteroid Bennu (Credit: NASA). 
During the TAG, SamCam, which is one of the three imagers of the OSIRIS-REx Camera Suite (OCAMS; PolyCam and MapCam are the other two), will continuously document the sample acquisition. ${ }^{4}$ There are three locations on the spacecraft that do not permit specular MLI materials or coatings. First, if the TAGSAM MLI outer cover, specifically on and around the nitrogen bottles, is specular, it will lead to sunlight glint into SamCam during the TAG and blind the imager. Second, if the PolyCam sunshade MLI is specular, it will reflect stray light into MapCam during the survey for dust hazards, which could be mistaken for dust. Third, if the MLI on the SamCam motor riser is specular, it will reflect stray light into SamCam at low Sun incidence angles. ${ }^{5,6,7}$

Figure 3 shows an approximate view of the TAGSAM from the SamCam during the TAG. Optical analysis showed that a diffuse coating or material is required for the MLI blankets on and around the TAGSAM arm nitrogen bottles. ${ }^{8}$ The bidirectional reflectance distribution function (BRDF) requirement is not to exceed $0.32 \mathrm{sr}^{-1}$ from $0^{\circ}$ to $50^{\circ}$ incidence angle. ${ }^{7}$

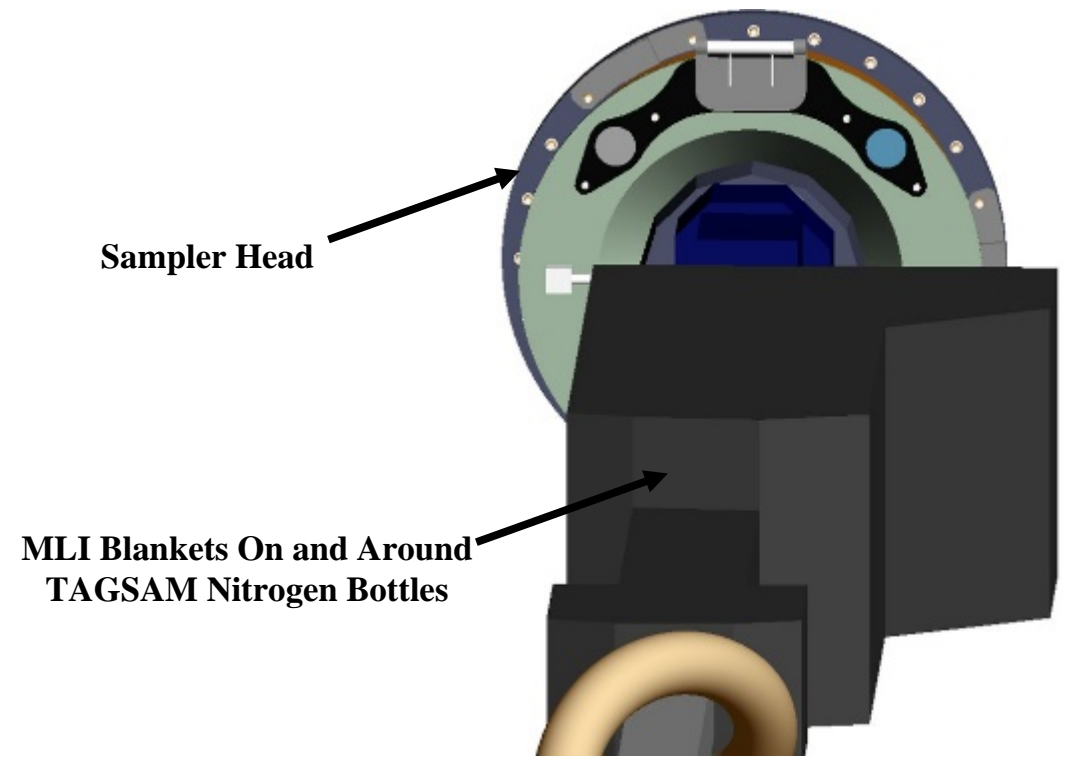

Fig. 3. Approximate view of TAGSAM from SamCam during TAG. ${ }^{8}$

To ensure that the asteroid sample does not overheat inside TAGSAM sampler head, the nominal mission design limits TAG to heliocentric distances beyond 1.25 AU. However, in an off-nominal situation, the mission team may decide to attempt sample acquisition inside this distance. Thus, during the TAG, it is possible that the TAGSAM MLI blankets have a long-term exposure to sunlight at a heliocentric distance of $0.897 \mathrm{AU}$. The solar irradiance is 1,724 $\mathrm{Wm}^{-2}$. The maximum predicted flight temperature of the MLI cover with black and diffuse materials in the stacked worst hot case is $153^{\circ} \mathrm{C}$. An absorptance of 0.96 and a hemispherical emittance of 0.89 are assumed. Additionally, it is assumed to be adiabatic. After adding a $10^{\circ} \mathrm{C}$ thermal design margin, the MLI blanket outer cover bakeout temperature to meet the mission contamination control requirement is $163^{\circ} \mathrm{C}$. The material must survive such a high temperature.

The PolyCam sunshade MLI also has long-term exposure to sunlight at a heliocentric distance of 0.773 to 1.39 AU in other mission phases. ${ }^{3}$ At $0.773 \mathrm{AU}$ in the hot cruise mission phase, the solar irradiance is $2,322 \mathrm{Wm}^{-2}$. The PolyCam sunshade is not adiabatic because its structure is sugar scoop shaped and is painted black on the interior. The black interior radiates heat to deep space through the aperture. The maximum predicted flight temperature for the microporous black PTFE in the stacked worst hot case is close to the $163^{\circ} \mathrm{C}$ bakeout temperature. ${ }^{9}$

All the MLI blanket outer cover materials commercially available today have high solar specularity. Kapton, black Kapton, germanium-coated black Kapton (GBK), StaMet-coated black Kapton, aluminum tape, and aluminum are all bright when they are exposed to the Sun. Black Kapton has a high solar absorptance, but its solar specularity is also high. BRDF measurement of black Kapton showed that it does not meet the SamCam BRDF requirement. ${ }^{9}$ We applied Aeroglaze Z307 diffuse black paint to black Kapton at NASA Goddard Space Flight Center (GSFC) to make the black Kapton diffuse. After a long bakeout at $163^{\circ} \mathrm{C}$ to meet the mission contamination control requirement, it failed because it flaked. 
Black and white microporous PTFE flexible thin sheets, which have no spaceflight heritage, were flightqualified at NASA GSFC for the OSIRIS-REx project. ${ }^{9}$ The size of the micropores in these PTFE thin sheets is approximately $2 \mu \mathrm{m}$. The thickness is $2.5 \mathrm{~mm}$. Manufacturing tools limited the widths of the black and the white PTFE thin sheets to 30 and $33 \mathrm{~cm}$, respectively. The black PTFE thin sheets are embedded with carbon black and are electrically conductive. The white PTFE thin sheets are pure PTFE, and are electrically non-conductive. Microporous black PTFE thin sheet meets the OSIRIS-REx SamCam BRDF requirement with plenty of margin. The contamination concern is low despite being non-zero. ${ }^{10}$ Microporous white PTFE thin sheet has zero contamination concern, but does not meet the SamCam BRDF requirement. ${ }^{9}$

\section{Use of Microporous Black PTFE Thin Sheets on OSIRIS-REx Flight Hardware}

The OSIRIS-REx spacecraft uses flight-qualified microporous black PTFE flexible thin sheets as solar diffusers. They are installed onto the TAGSAM MLI blanket (Figure 4), PolyCam sunshade MLI blanket (Figures 4 and 5), and SamCam motor riser MLI blanket (Figures 4 and 6). The 3M 9082 high-temperature acrylic transfer adhesive bonds the microporous black PTFE thin sheets to the MLI outer layer. The thin sheets were also stitched to the TAGSAM MLI with diffuse fiberglass threads to add fastening. The surface area of the stitches on the exterior of the microporous black PTFE and within the field of view (FOV) of the SamCam is very small. They are expected to have a relatively limited and mostly cosmetic imaging impact on the SamCam.

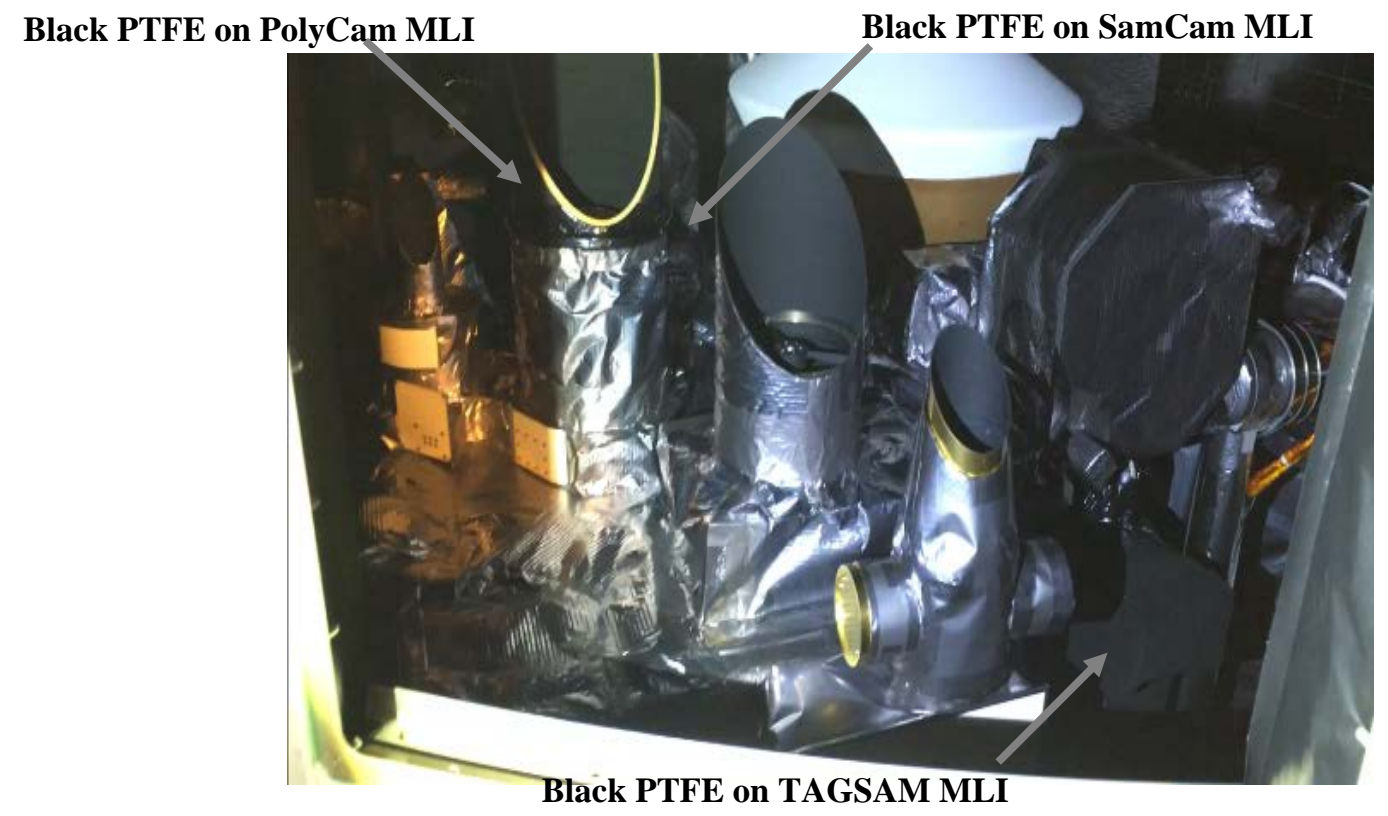

Fig. 4. Microporous black PTFE on OSIRIS-REx flight hardware (Credit: NASA GSFC).

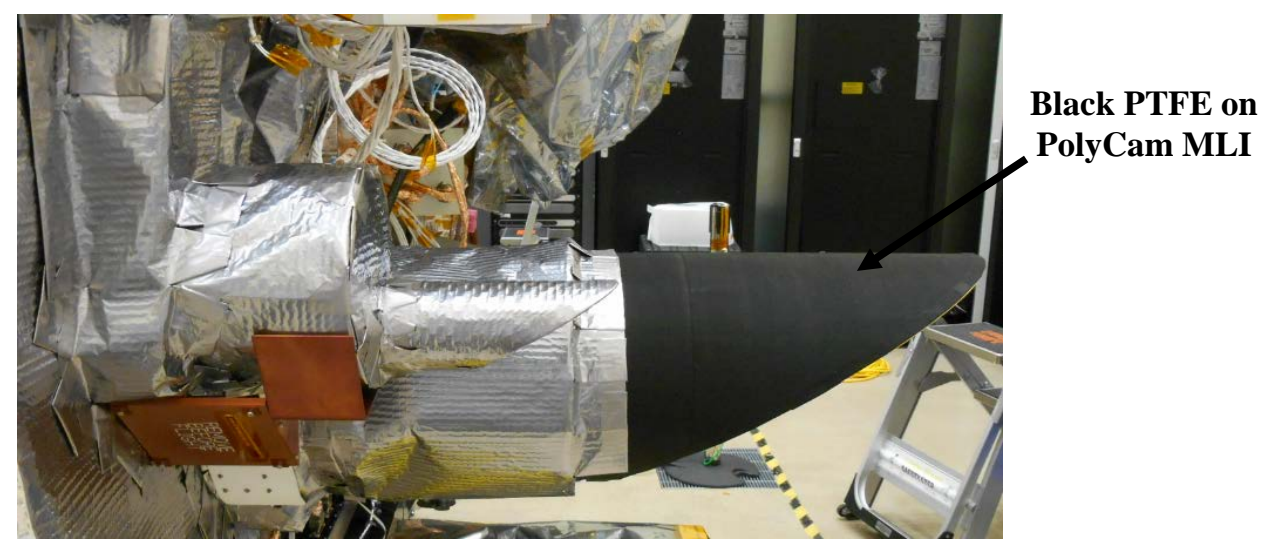

Fig. 5. Microporous black PTFE on PolyCam MLI (Credit: NASA/Goddard/University of Arizona). 


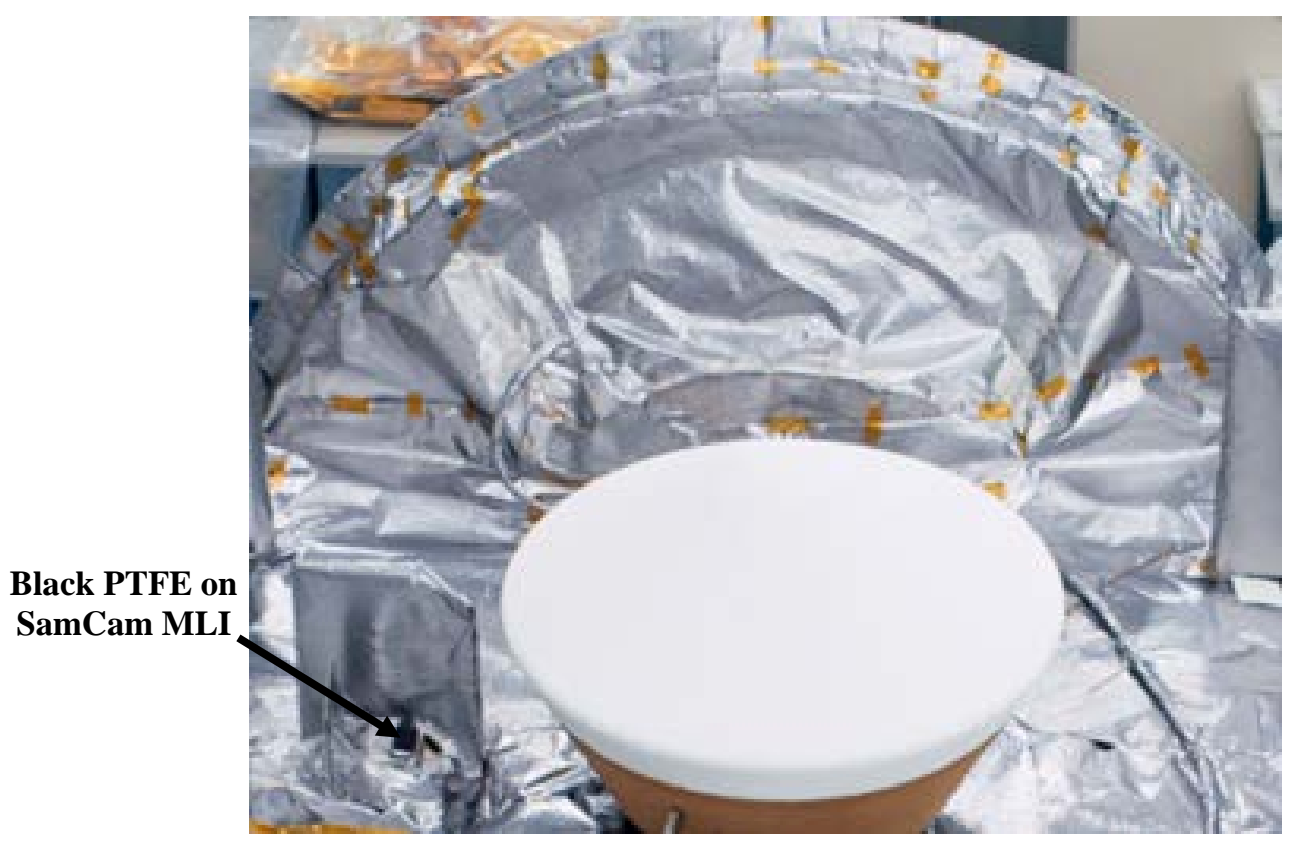

Fig. 6. Microporous black PTFE on SamCam MLI (Credit: NASA).

Figure 7 shows a view of the microporous black PTFE on the TAGSAM MLI blanket immediately after the spacecraft separation from the launch vehicle. Its diffuse black appearance in sunlight contrasts sharply with the specular GBK MLI blankets on the bulk of the spacecraft.

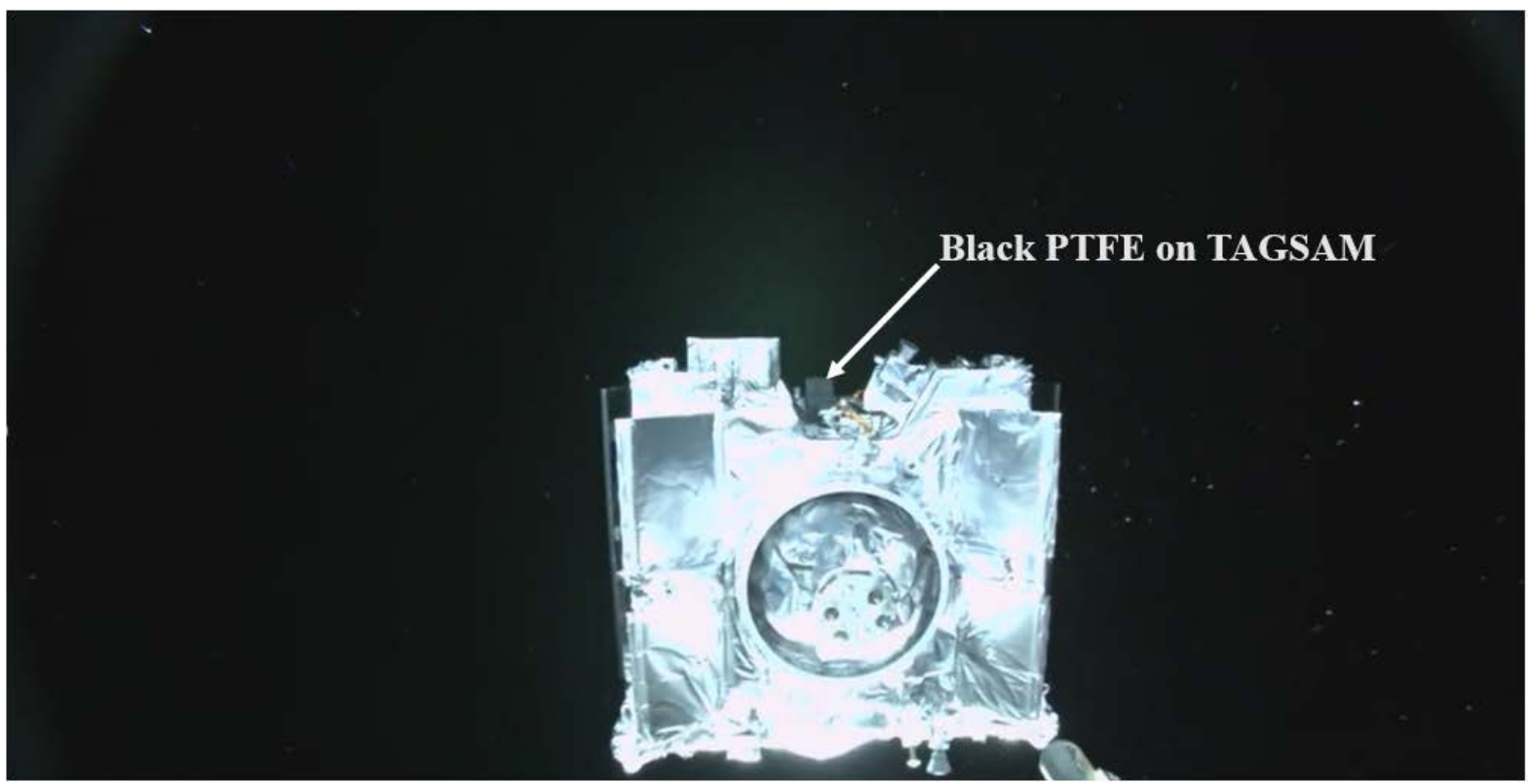

Fig. 7. View of microporous black PTFE on TAGSAM during launch (Credit: NASA).

SamCam acquired images of the TAGSAM on November 14, 2018. There was no sunlight or stray light glint into SamCam. Figure 8, an example of those images, shows the microporous black PTFE on the TAGSAM MLI blanket. The location of the black PTFE can be compared to that in Figure 3, which was used in the OSIRIS-REx design phase. Sunlight impinged on the backside of the sampler head and illuminated it. In contrast, in Figure 8, the microporous black PTFE looks diffusely black. It satisfies the SamCam stray light requirement. 


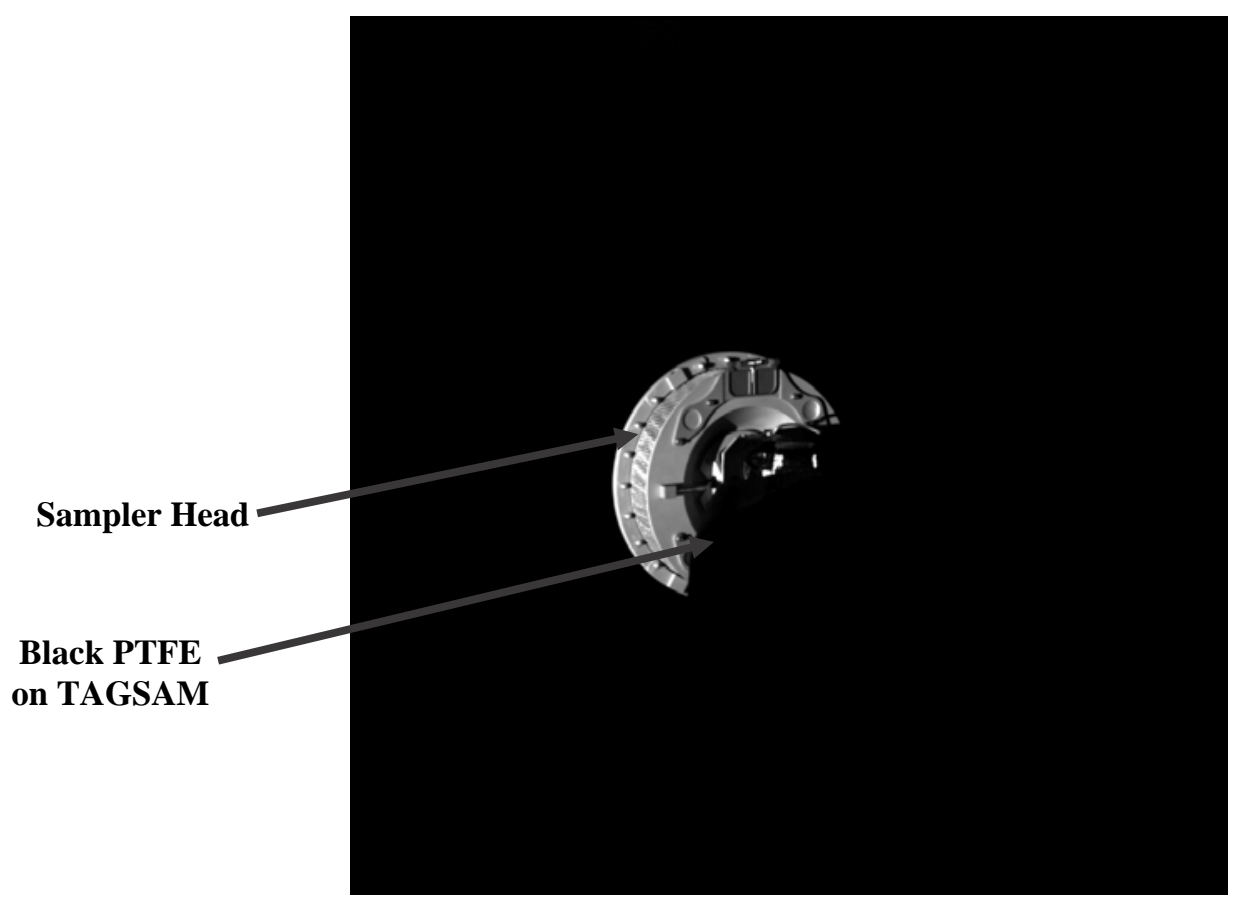

Fig. 8. SamCam view of TAGSAM on November 14, 2018 (Credit: NASA).

MapCam acquired images of space surrounding asteroid Bennu on September 12, 2018, during the OSIRIS-REx mission's search for dust plumes. No sunlight or stray light glint was reflected by the PolyCam sunshade MLI blanket into MapCam. The microporous black PTFE cover on the PolyCam sunshade MLI blanket meets the MapCam stray light requirement.

\section{Use of Microporous White PTFE Thin Sheets on OSIRIS-REx Flight Hardware}

The Regolith X-ray Imaging Spectrometer (REXIS) will perform science operations in the Orbital B phase of the OSIRIS-REx mission. ${ }^{11}$ Its charge coupled device (CCD) detector requires an operational temperature in the range of $-120^{\circ} \mathrm{C}$ to $-60^{\circ} \mathrm{C} \cdot{ }^{12}$ It is cooled passively by a radiator with Z93C55 white paint on the $+\mathrm{Y}$ side. The detector plate is thermally isolated from the REXIS electronics box, which is mounted to the payload $(+Z)$ deck structure through a mounting flange. Except for the radiator white paint, coded mask, and mask frame, its exterior is insulated with MLI blankets. During Orbital B, the spacecraft is $20^{\circ}$ off-point. The MLI blankets on the REXIS deployed radiation cover and $+X$ and $-Y$ sides are exposed to direct sunlight. Its MLI blankets on the $+X,-X$, and $-Y$ sides also receive sunlight reflected or scattered by other MLI blankets on the payload deck.

NASA GSFC was responsible for the design, fabrication, and integration of the REXIS MLI blankets. Microporous white PTFE flexible thin sheets were used as thermal covers for the exterior of the REXIS MLI blankets. The MLI blanket exterior has a low solar absorptance and a high emittance. Its measured absorptance is 0.085 at the beginning of life, which increased to 0.3 at the end of life after the solar wind test at NASA GSFC. ${ }^{9}$ Its hemispherical absorptance with an aluminized Kapton substrate is 0.87 .

Microporous white PTFE is electrically non-conductive. The smaller its size, the lower the electrostatic discharge (ESD) concern. The area of the radiation cover MLI blanket is $0.019 \mathrm{~m}^{2}$. A microporous white PTFE thin sheet covers the entire area. The largest REXIS MLI blanket is $0.103 \mathrm{~m}^{2}$ and wraps around the $+\mathrm{X},-\mathrm{X}$, and $-\mathrm{Y}$ sides. A separate patch $\left(0.0343 \mathrm{~m}^{2}\right)$ of microporous white PTFE flexible thin sheet covers each side to minimize the ESD concern. The 3M 97057 conductive acrylic transfer adhesive bonds the microporous white PTFE thin sheets to the aluminized Kapton outer layer of the MLI blankets. NASA GSFC's flight heritage Cho-foil aluminum grounding tapes ground the PTFE to the MLI blanket. The first grounding tape runs along one edge of each PTFE patch. Except for a small area $(1 \mathrm{~cm} \mathrm{x} 1 \mathrm{~cm})$, it is covered by Kapton tape by using the 3M 97057 conductive acrylic transfer adhesive. The second grounding tape grounds the first one to the MLI blanket ground wire. The ground wire is in turn grounded to the flange mounting the REXIS electronics box to the payload deck. This grounding scheme allows the microporous white PTFE flexible thin sheet to bleed charges. Kapton tape, with the 3M 97057 conductive acrylic transfer adhesive, 
runs along the other edges of the white PTFE to add fastening to the structure. Figure 9 shows the REXIS MLI blankets with microporous white PTFE flexible thin sheets as the outer covers.
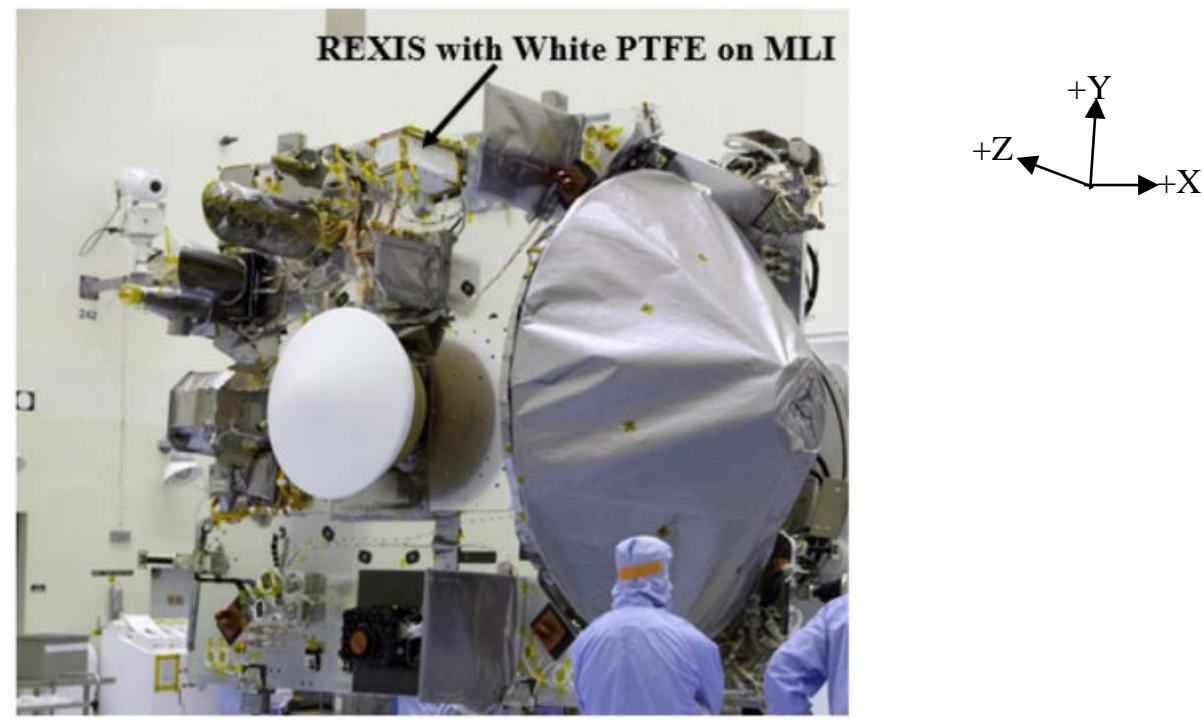

Fig. 9. View of white PTFE on REXIS MLI (Credit: NASA).

The steady-state REXIS operating detector temperature was $-92^{\circ} \mathrm{C}$ at a heliocentric distance of $1.1 \mathrm{AU}$ after the radiation cover was deployed. The spacecraft attitude was nominal Sun-point (i.e., $0^{\circ}$ off-point). Sunlight impinged on part of the radiation cover at that attitude. During Orbital B, the spacecraft is $20^{\circ}$ off-point, meaning that sunlight impinges on the microporous white PTFE on the entire radiation cover and $+X$ and $-Y$ sides. The operating CCD detector temperature is expected to be well below the $-60^{\circ} \mathrm{C}$ maximum allowable flightlimit.

\section{Use of Microporous PTFE Thin Sheets for TAGCAMS Optical Targets in Flight System}

The Touch And Go Cameras System (TAGCAMS) includes two NavCam imagers. Figure 10 shows the locations of these imagers. An objective of the OSIRIS-REx flight system thermal vacuum (TVAC) test was to acquire TAGCAMS images for assessing the NavCam image quality and pointing stability. An optical target was required for each of the two NavCam imagers. ${ }^{13}$

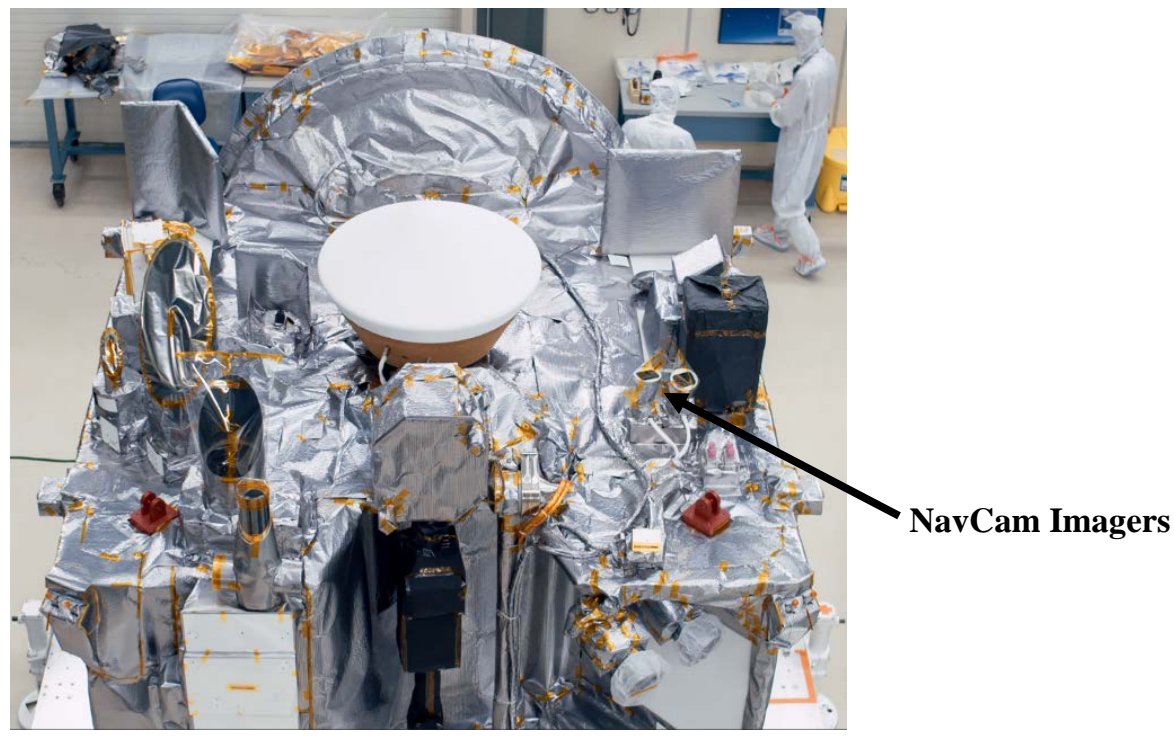

Fig. 10. Locations of TAGCAMS NavCam imagers (Credit: NASA). 
The optical targets were fabricated at NASA GSFC. Two layers of microporous white PTFE flexible thin sheet were used as the background. The first $60.96 \mathrm{~cm}$ x $60.96 \mathrm{~cm}$ layer was bonded to an aluminum plate by the 3M 97057 conductive acrylic transfer adhesive. As mentioned earlier, the width of the microporous white PTFE flexible thin sheets was limited by manufacturing tools to $33 \mathrm{~cm}$. Therefore, a 33-cm-wide white PTFE was used in the middle of the optical targets, and a 13.98-cm-wide white PTFE was used on each side. The second 60.96 $\mathrm{cm}$ x $60.96 \mathrm{~cm}$ layer was bonded to the first layer by the above adhesive. A $30.48 \mathrm{~cm}$ x $30.48 \mathrm{~cm}$ microporous black PTFE flexible thin sheet, centered on the target, was bonded to the white PTFE by the above adhesive. The black PTFE thin sheet had eight 00.635 -cm-diameter holes, which are equally spaced at $7.62 \mathrm{~cm}$ and penetrate through to the white PTFE. ${ }^{13}$ They are shown in Figures 11 and 12 for targets 1 and 2, respectively. The optical targets were included in the vacuum chamber bakeout prior to the flight system TVAC test at Lockheed Martin in Denver.

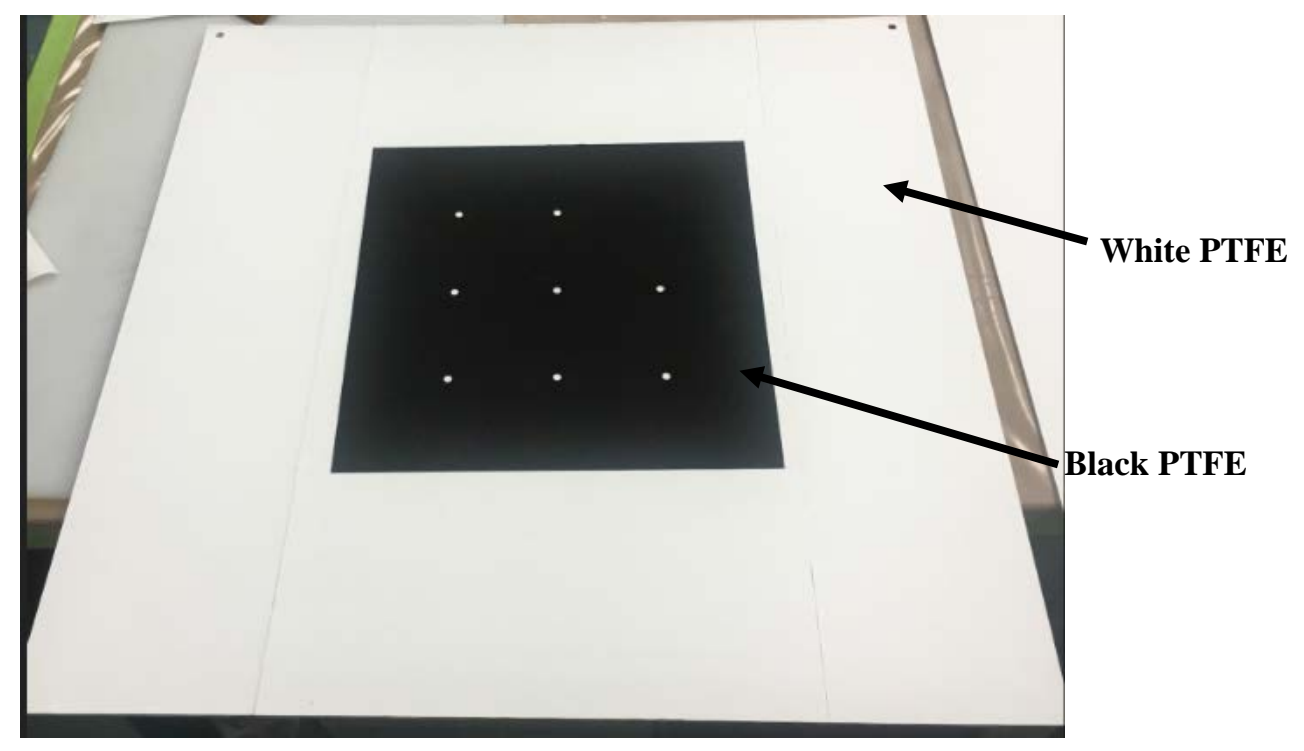

Fig. 11. TAGCAMS NavCam 1 optical target.

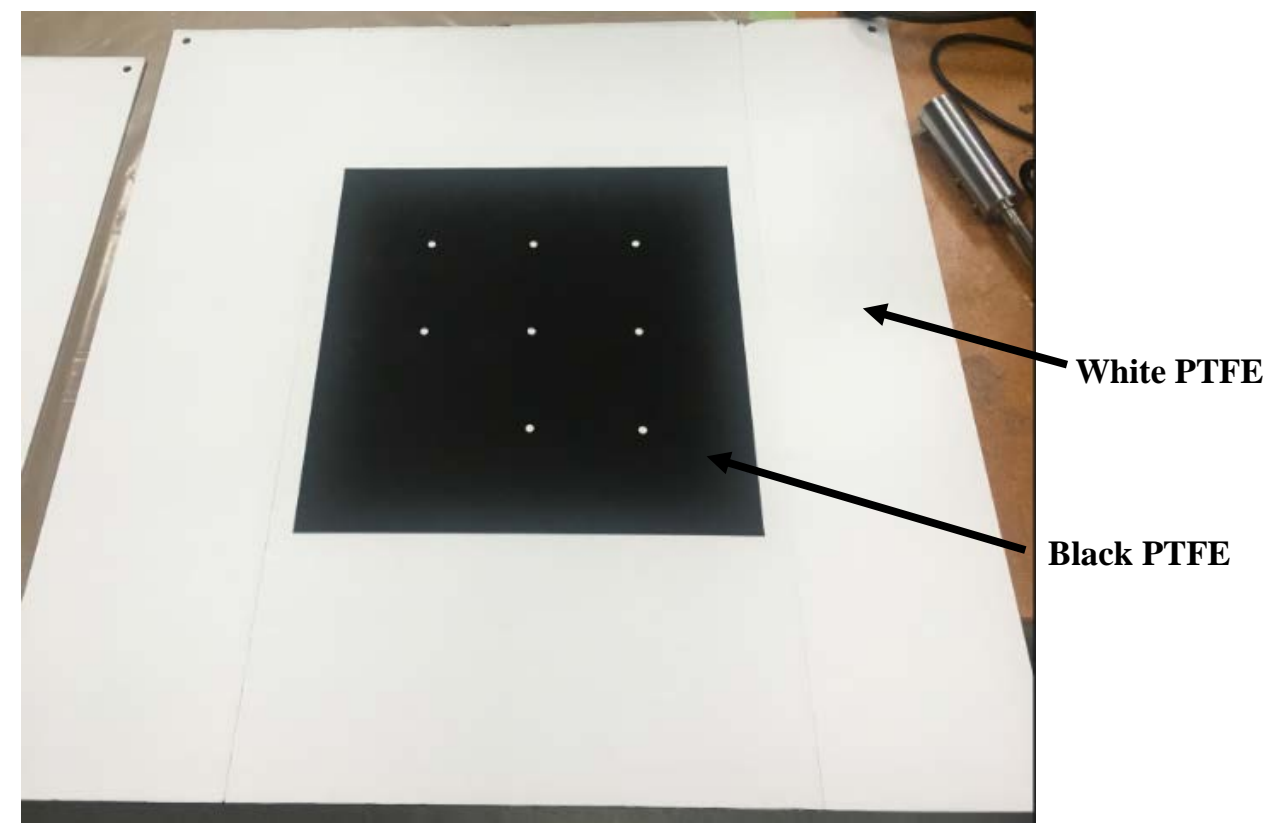

Fig. 12. TAGCAMS NavCam 2 optical target. 
The NavCam optical targets were suspended in the vacuum chamber as shown in Figures 13 and 14 . The vacuum chamber shroud temperature data in the TVAC versus time is shown in Figure 15 . The temperature was $-170^{\circ} \mathrm{C}$ for about ten days and between $-100^{\circ} \mathrm{C}$ and $-60^{\circ} \mathrm{C}$ for about five days. The temperatures of the NavCam optical targets were very close to that of the chamber shroud. The PTFE thin sheets had good adhesion for the entire TVAC test. TAGCAMS images were successfully acquired for assessing the NavCam image quality and pointing stability.

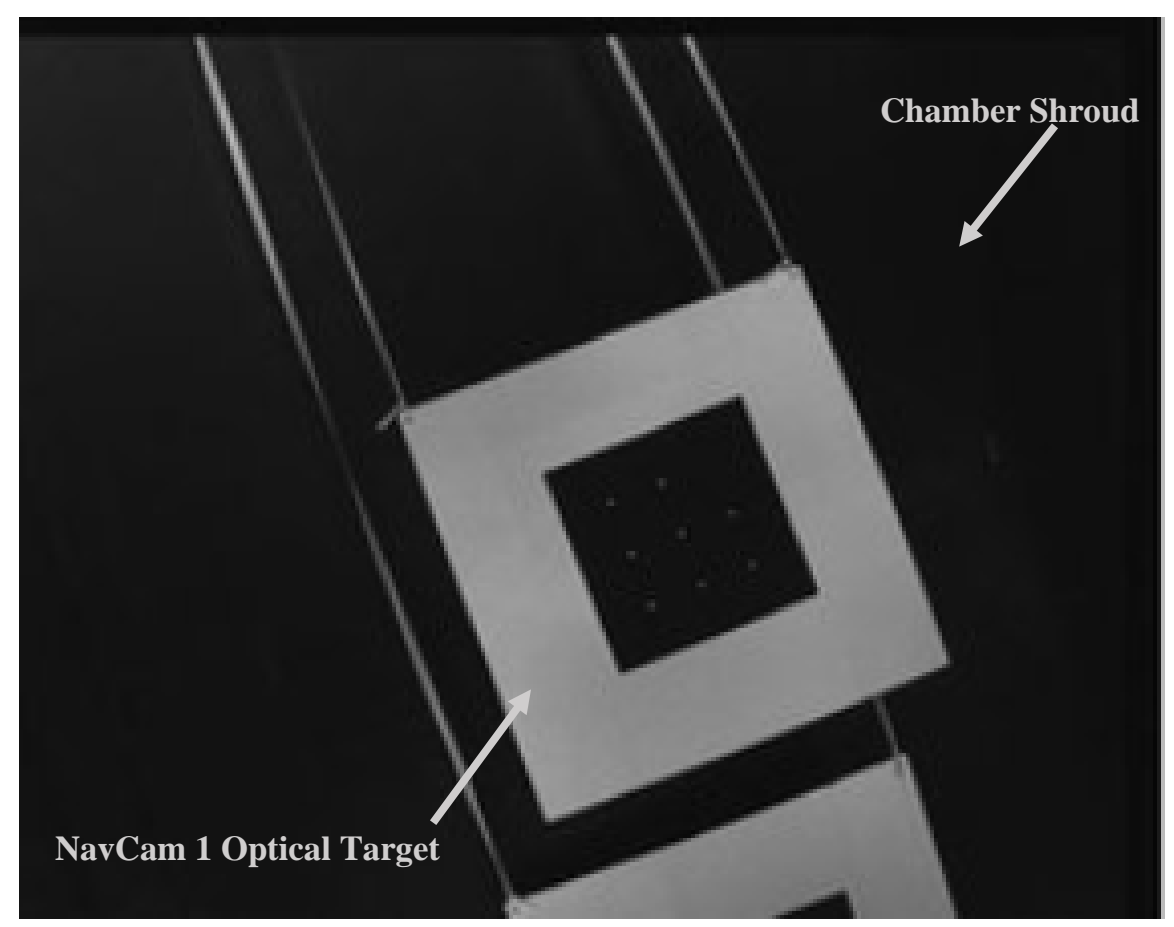

Fig. 13. TAGCAMS NavCam 1 optical target in vacuum chamber. ${ }^{13}$

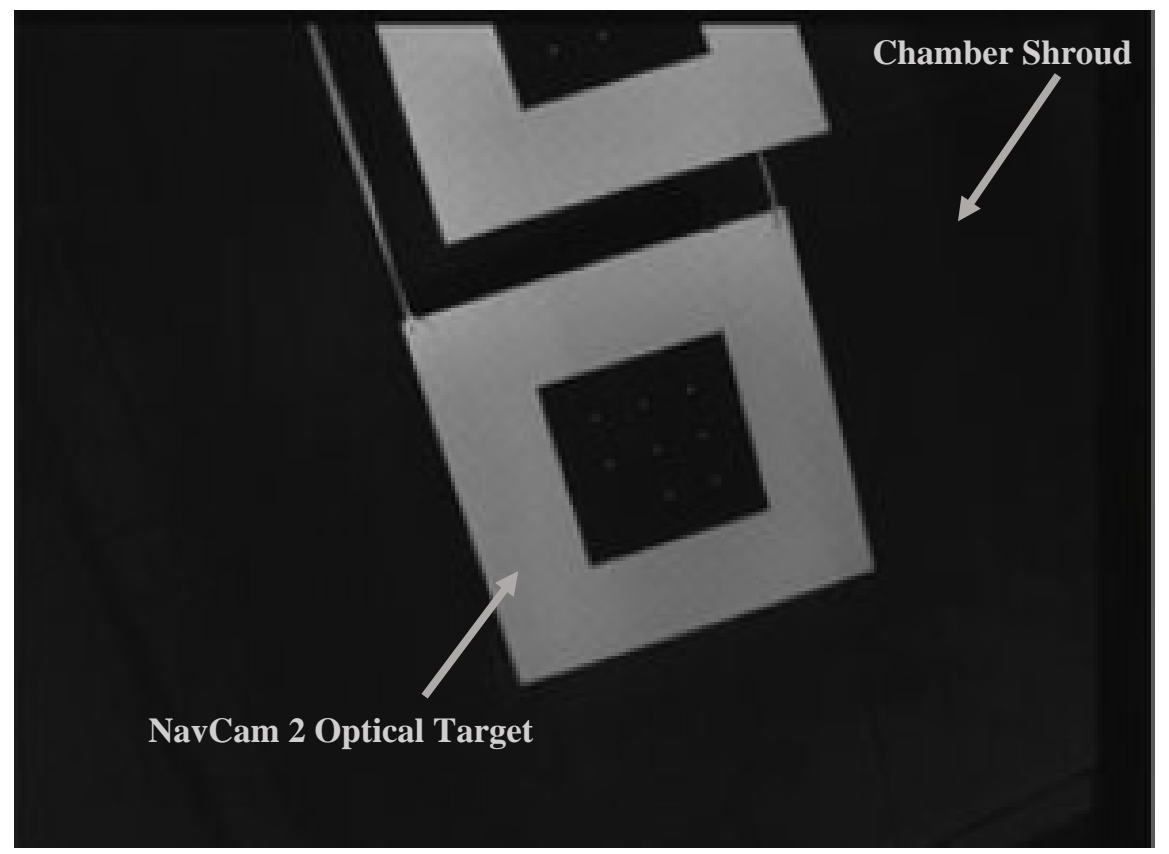

Fig. 14. TAGCAMS NavCam 2 optical target in vacuum chamber. ${ }^{13}$ 


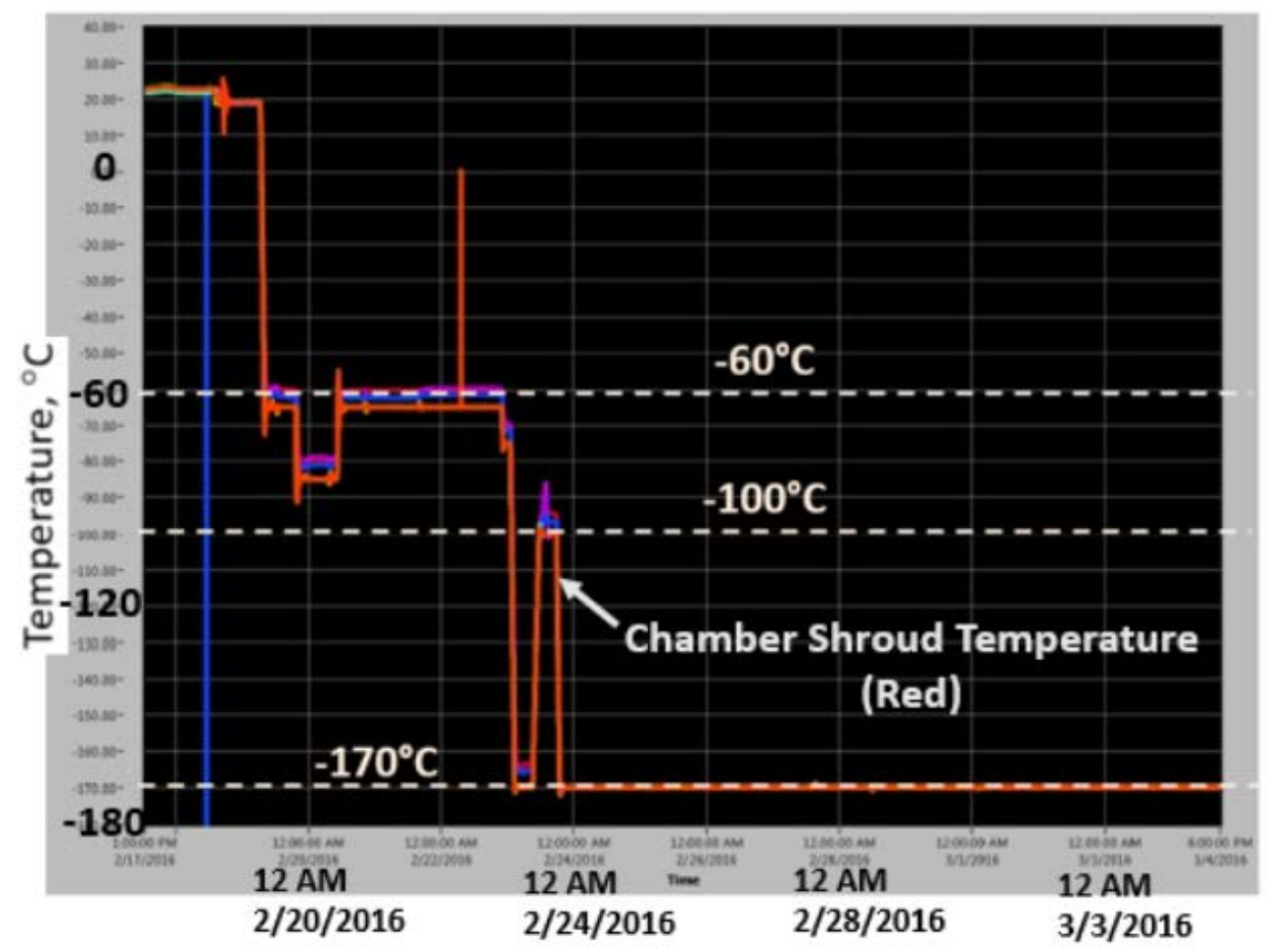

Fig. 15. Vacuum chamber shroud temperature versus time during flight system TVAC.

\section{Conclusion}

Microporous PTFE flexible thin sheets of $2.5 \mathrm{~mm}$ thickness are successfully flown in NASA's OSIRIS-REx mission. This is the first time that microporous PTFE flexible thin sheets have been used on a spacecraft. Microporous black PTFE flexible thin sheets serve as solar diffusers for the MLI blankets on three spacecraft components: the TAGSAM arm, the OCAMS PolyCam sunshade, and the OCAMS SamCam motor riser. The 3M 9082 hightemperature acrylic transfer adhesive bonds them to the MLI blanket outer layers. Because the TAGSAM MLI blanket is like a large bag, microporous black PTFE thin sheets are also stitched to the MLI blanket by diffuse fiberglass threads to add mechanical fastening. Microporous white PTFE thin sheets serve as an outer cover with a low ratio of absorptance to emittance for the REXIS MLI blankets. Both black and white microporous PTFE flexible thin sheets were successfully used for the TAGCAMS NavCam optical targets in the flight system TVAC test. SamCam acquired images of the TAGSAM during OSIRIS-REx's approach to Bennu. They include a view of the microporous black PTFE solar diffuser on the TAGSAM MLI blanket. The microporous white PTFE flexible thin sheets on the REXIS instrument reduce the thermal effect of solar impingement on its MLI blankets. A reduction in parasitic heat load to REXIS's CCD through the MLI blankets allows its CCD temperature to meet the requirement. NASA's OSIRIS-REx mission demonstrates that microporous PTFE flexible thin sheets can be used not only as solar diffusers but also for thermal management - for example, as thermal radiators or diffuse black enclosure for optics. If necessary, fasteners can be added to better attach them to the mechanical structure.

\section{Acknowledgments}

This material is based upon work supported by NASA under Contract NNM10AA11C issued through the New Frontiers Program. The successful use of microporous PTFE flexible thin sheets was brought about by the government and industry team work. The authors acknowledge the following individuals for their excellent work in installing the microporous PTFE flexible thin sheets to the flight hardware: Christian May and Jeremy Bennington of Lockheed Martin Space System Company. The authors also thank the following individuals for their technical contributions during the development phase: David Everett and Gary Davis of the GSFC Mission Systems Engineering Branch; Charles Lorentson, Griffin Jayne, and Lon Kauder of the GSFC Contamination and Coatings Engineering Branch; Christian May and Jeremy Bennington of Lockheed Martin Space System Company. Berghof Fluoroplastic 
Technology GmbH supplied the microporous black PTFE flexible thin sheet, and Porex Corporation supplied the microporous white PTFE flexible thin sheet. Their assistance to deliver their products on time to meet the extremely tight flight qualification and testing schedule at GSFC is much appreciated.

\section{References}

[1] Lauretta, D. S., et al. "OSIRIS-REx: sample return from asteroid (101955) Bennu." Space Science Reviews 212.12 (2017): 925-984.

[2] Bierhaus, E. B., et al. "The OSIRIS-REx flight system and Touch-and-Go Sample Acquisition Mechanism." Space Sci. Rev (2018).

[3] Linn, T., et al., "TAGSAM, 1_2 - TAG Event Summary", OSIRIS-REx SARA/TAGSAM/SRC CDR, LMSSC (36 February 2014) (unpublished).

[4] Rizk, B., et al. "OCAMS: the OSIRIS-REx camera suite." Space Science Reviews 214.1 (2018): 26.

[5] Linn, T., et al., "TAGSAM, 1_2 - TAG Event Summary", OSIRIS-REx SARA/TAGSAM/SRC CDR, LMSSC (36 February 2014) (unpublished).

[6] Sutter, B., "Possible Sun Angles During TAG vs. Latitude on Asteroid", LMSSC, email to OCAMS team (13 February 2014) (unpublished).

[7] D’Aubigny, C. and Pfisterer R., "SamCam TAGSAM Glint Evaluation”, University of Arizona (18 June 2014) (unpublished).

[8] Tveekrem, J., "Sun Glint into SamCam from TAGSAM Wrist MLI, Rev. 1", OSIRIS-REx Project, GSFC (12 September 2015) (unpublished).

[9] Choi, M. K., "Using microporous polytetrafluoroethylene thin sheets as a flexible solar diffuser to minimize sunlight glint to cameras in space”, Proc. SPIE 9981-13 (2016).

[10] Dworkin, J. P., et al., "OSIRIS-REx Contamination Control Strategy and Implementation”, Space Science Reviews (2018) 214:19.

[11] Masterson, R. A., et al. "Regolith X-Ray Imaging Spectrometer (REXIS) Aboard the OSIRIS-REx Asteroid Sample Return Mission." Space Science Reviews 214.1 (2018): 48.

[12] Stout, K., "REXIS Critical Design Review: Thermal System”, REXIS Critical Design Review: February 18-20, 2014 (unpublished).

[13] Bos, B. J., et al., "Touch And Go Camera System (TAGCAMS) for the OSIRIS-REx Asteroid Sample Return Mission”, Space Science Reviews (2018) 214:37. 